\title{
Simple rotary motion is integrated across fixations
}

\author{
ALEXANDER POLLATSEK and KEITH RAYNER \\ University of Massachusetts, Amherst, Massachusetts
}

\begin{abstract}
Participants were shown a line rotating at a constant angular velocity and were asked to judge whether motion was continuous or whether the line jumped (i.e., moved either forward or backward in the rotary cycle). In two experiments, the participants were significantly better than chance in detecting these jumps in simple rotary motion even when the jumps occurred during a saccade. Moreover, in Experiment 2, when the jump occurred during a saccade followed by a masking flash, perception of the jump was at least as good as when it occurred during a fixation followed by a masking flash. These results complement and extend the findings of Verfaillie and co-workers, who found that perceptions of some changes in biological motion were as good across fixations as when they occurred during a fixation. These findings are in contrast to the common finding, in the reading literature, that people are consciously "blind" to many changes in the text and to those, in the scene perception literature, in which detection of static changes across fixations is above chance but plausibly well below the level that would be expected if the change occurred during a fixation.
\end{abstract}

During the usual viewing of a static scene, we make about three saccadic eye movements every second (Henderson \& Hollingworth, 1998; Rayner, 1998). This is surprising to most people, since they are usually unaware of the frequency of their eye movements and their introspective experience is one of perceiving the world uninterruptedly and forming a coherent integration of the visual information in the scene over time. Instead, viewers of a scene are getting a series of "snapshots" of a visual display, out of which they are somehow making continuous sense. A major question in vision is what kinds of information are being maintained, from a prior fixation, that are used somehow in forming the visual percept that extends over time (Pollatsek \& Rayner, 1992).

Two basic types of paradigms have been employed to investigate the types of information that are maintained across fixations (see Rayner, 1998, for a review of these paradigms). The first involves making a change in a scene during a saccade (when vision is suppressed) and determining whether this change is noticed. The second involves trying to determine whether information obtained on one fixation aids in processing the information on subsequent fixations (i.e., whether information is integrated

This research was supported by Grant HD26765 from the National Institute of Health, by KDI Grant 9980045 from the National Science Foundation, and by a grant from the General Electric Fund. The second author was also supported by a Research Scientist Award from the National Institute of Mental Health (MH01255). We thank Helene O'Connor for assistance in collecting the data and John Henderson, Dan Simons, Karl Verfaillie, and an anonymous reviewer for their helpful comments on an earlier version of the article. Correspondence concerning this article should be sent to A. Pollatsek, Department of Psychology, University of Massachusetts, Amherst, MA 01003 (e-mail: pollatsek @ psych.umass.edu). across fixations). Typically, a display change is made across fixations, and one determines whether identification of an object is interfered with when this change is made and what kinds of changes affect identification.

Our interest in this issue arose from our work in reading because, during reading, people have little awareness of fairly major changes in the display across saccades (see Rayner, 1998, for a review of this research). In these experiments, text is presented on a screen (with a window of normal text around the fixation point), and a display change is made during a saccade. People are unaware of display changes and are unaware of there being anything other than normal text on the screen when (1) the window contains the fixated word and the first three letters of the next word, (2) the lengths of the "words" outside the window are held constant across saccades, and (3) the strings of letters outside the window are reasonably textlike. This is true even when display changes occur on every saccade (Rayner, Well, Pollatsek, \& Bertera, 1982). Some physical changes are detected across fixations, such as changes from uniform strings of $x \mathrm{~s}$ to letters or changes in the sizes of words, but people are unaware when a "word" changes from a string of random letters to a word across a saccade. There are also other low-level physical changes that participants appear to be relatively "blind" to. For example, when the whole line of text is moved during a saccade, it has to be moved more than 2-3 characters before the move is noticeable (McConkie, Zola, \& Wolverton, 1980). Moreover, when participants detect this change, it is not clear whether they are detecting the change per se or whether they are merely noticing that they are not fixating on the word that they intended to fixate. ${ }^{1}$

In addition, research in reading indicates that there is little visual information retained from one saccade that 
helps to subserve identification of words on later fixations. Perhaps the two most striking findings in this regard are those of Rayner, McConkie, and Zola (1980) and McConkie and Zola (1979). In the former experiment, participants saw a parafoveal preview of a single word. When the word appeared in the parafovea, they moved their eyes to fixate the word and named it. The key finding was that the naming time was not any longer when the case of all the letters in the word changed during the saccade than when the stimulus remained constant across the saccade. One might argue that this result is an artifact of the naming task and that participants might have been insensitive to the display change because a nonvisual level of coding was tapped to produce the name. However, McConkie and Zola obtained a similar result when participants read sentences in aLtErNaTiNg CaSe. In one condition, the text remained constant throughout the trial, whereas in another condition, the case of every letter changed on each fixation (e.g., aLtErNaTiNg CaSe would change to AlTeRnAtInG cAsE). They also found that the change of case had no effect. It thus appears that the level of featural processing in which upper- and lowercase letters differ is apparently not used in integrating information across saccades in reading. Note that there is often little physical similarity between the upper- and the lowercase versions of many letters (e.g., aA, nN).

Thus, the details of the visual features of words do not appear to be maintained across fixations either to allow one to notice that a change has been made or to help identify the word when it is later fixated. One might argue that these results are an artifact of the reading task. That is, the stimuli are not "natural," and furthermore, these arbitrary symbols are presumably being quickly recoded into various linguistic codes. Nonetheless, if integration of visual information (e.g., the features of objects) across saccades is a general mode of perception, it is not clear why it would be turned off during the reading task and, hence, why the change of features during a case change would not be disruptive.

An interesting question, of course, is what kinds of information in more natural visual (i.e., nonreading) situations are extracted from one fixation and used on the next fixation. The problem, of course, is that unlike with symbolic stimuli, it is quite difficult to separate out visual levels of processing from deeper, semantic levels of processing. Thus, if participants notice that a stimulus has changed from a dog to a horse across fixations, it is not clear whether this is because they have noticed the visual mismatch or because the mismatch is coded at a different level (e.g., they noticed the meaning change or the name change). In an attempt to sort out these levels, Pollatsek, Rayner, and Collins (1984) employed line drawings of objects in a naming task analogous to the experiment of Rayner et al. (1980) discussed above, in which a parafoveal preview was provided. Pollatsek et al. attempted to distinguish between these levels by using pairs of pictures (i.e., the preview seen in the parafovea before the saccade and the target seen in the fovea after the saccade) that were either visu- ally similar or semantically similar and examined whether there was greater benefit on naming latency from the preview when it was visually similar to the target. Significant effects of visual similarity (e.g., the preview was a carrot and the target was a baseball bat) on preview benefit occurred only when the preview was far $\left(10^{\circ}\right)$ from fixation. Because this visual similarity effect occurred only when the preview was far from fixation, it could merely reflect those trials in which the parafoveal object was misidentified as the target object (e.g., the carrot was misidentified as a baseball bat). ${ }^{2}$

Pollatsek et al. (1984) also employed an analogue of the case change manipulation by changing the size of the object $10 \%$ from fixation to fixation. Analogous to the case change experiments in reading, performance in these conditions was the same as when there was no change from the parafoveal stimulus to the foveal stimulus (see also Henderson, Pollatsek, \& Rayner, 1987). One physical change that did seem to make a significant difference was when the object seen in the fovea (following a saccade) was a mirror image of the one seen in the parafovea. In this condition, naming time was $37 \mathrm{msec}$ slower than in the condition in which the parafoveal and the foveal stimuli were identical; however, the difference was only marginally significant. The latter result has been extended by Henderson and Siefert (1999), who found that changes in the viewpoint of an object across fixations significantly affected both naming time for individual objects and the gaze duration on the object when it was in an array of four objects that were examined (followed by a short-term memory probe).

In another type of visual integration task that has been extensively studied, participants see a collection of tokens (e.g., dots) that occupy random positions in a fixed matrix on one fixation, and a second set of (nonoverlapping) tokens appears on the following fixation, and then the participant is asked to make a judgment about the combination of the two set of tokens. For example, both the first fixation and the second fixation may each contain 12 dots, with each dot filling up a slot in a $5 \times 5$ matrix; the viewer is then asked to decide which slot of the matrix had the missing dot. The finding with arrays of this size (Irwin, Yantis, \& Jonides, 1983; Rayner \& Pollatsek, 1983) was that performance was only marginally above chance when screen persistence of the image from the first fixation was eliminated, and it was concluded that there was little or no integration of low-level visual information across fixations. However, substantially better than chance performance was found when the matrix was smaller (e.g., a $3 \times 3$ matrix). This pattern of data is consistent with a short-term memory interpretation. That is, when there are few dots, it should be fairly easy to code the positions of most of them, relative to the matrix frame in a more abstract form (perhaps verbal), and to use those codings to evaluate where the missing dot is (Irwin, 1992; see also Hayhoe, Lachter, \& Feldman, 1991).

To summarize, the experiments on reading indicate that there is little or no information that could be termed visual 
that is preserved across a saccade that is of service in identifying the "objects" (i.e., words). Instead, it appears that the information is preserved from fixation to fixation either as "abstract letter identities" (Rayner et al., 1980) or as phonologicalcodes (Pollatsek, Lesch, Morris, \& Rayner, 1992). It does appear that some changes of visual information are registered, such as where the spaces between words are (Morris, Rayner, \& Pollatsek, 1990; Pollatsek \& Rayner, 1982). However, even in this regard, it is worth noting that Pollatsek, Raney, LaGasse, and Rayner (1993) found no detrimental effect in reading in a condition in which the text below the line that was being read was text from a completely different paragraph and, hence, the spaces between words were virtually all in the "wrong" places (some of them quite close to fixation). In contrast, in picture naming, at least, there seems to be some evidence that visual information is being stored, across saccades, that helps in the identification of objects (Pollatsek et al., 1984). However, even here, the effects are modest (about $30 \mathrm{msec}$ ) and not entirely consistent.

Most of the above research phrased the question of whether visual information is being used across fixations in terms of integration - that is, whether visual information extracted on one fixation helps one identify something better or more effectively on the subsequent fixation. A second line of research has emerged (which was briefly touched on above), in which a different probe is used: Participant are asked whether anything has changed from fixation to fixation. For many nonverbal situations, this is often an easier question to pose and is the paradigm employed in the present experiments. However, it taps a different processing question than the question of integration. One could also argue that it is somewhat less ecologically valid than the integration question, since the world rarely changes while a saccade is being made and, thus, it is hard to see what real-world task this would be relevant to (unlike reading or objectidentification). Nonetheless, it is of interest to see what participants do notice about changes in a display across fixations when they are looking for them.

One variant of the change detection paradigm is the following. A scene is presented, and then, on a particular saccade, there is a change in the scene; then, on a later saccade, there is a second change, which is back to the original scene (e.g., McConkie \& Currie, 1996; see, also, Currie, McConkie, Carlson-Radvansky, \& Irwin, 2000; Irwin, McConkie, Carlson-Radvansky, \& Currie, 1994). (On control trials, of course, there is no change.) The change is, typically, a change of an object in the scene, the deletion of an object from the scene, or a substantial change in the location of an object in the scene. The major finding from these experiments is that participants often do somewhat better than chance but perform quite poorly (the correct detection of change is often around 10\%-15\%) even though some of the changes made from fixation to fixation are quite large. Similar findings in a slightly different paradigm have been reported by Carlson-Radvansky (1999) and Carlson-Radvansky and Irwin (1995). ${ }^{3}$
In many of these experiments, however, the object that was changed was often far from both the fixation before the saccade and the fixation after the saccade. Thus, failure to detect the change may have been due merely to the fact that the visual information was poorly encoded either before or after the change or both. To remedy this problem, a new paradigm introduced by Henderson and Hollingworth (1999) made the display change contingent on where the participant's eyes were in the scene. In particular, the display change was made either (1) when the participant fixated a target object and the saccade following this fixation left the target object region or (2) when a saccade came to the target object region from outside the target object region. There were two types of changes: Either the target object was deleted following the saccade, or the target object was rotated $90^{\circ}$ in the horizontal plane (e.g., the target object was a clock sitting on a table, which was either deleted or rotated $90^{\circ}$ on the table). They found quite good performance on the deletion detection task (almost $80 \%$ in most conditions), whereas performance in the rotation detection task was considerably poorer (about $25 \%$ ) but still well above the false alarm rate (about 3\%). Several other analyses indicated that the distance of the target object from either of the fixation locations generally had a large influence on the probability of detecting the change.

One paradigm, however, shows amazingly good retention of what would naturally be called visual information across fixations. In this paradigm (Verfaillie, De Troy, \& Van Rensbergen, 1994), participants saw a set of moving dots on the screen that represented a person walking. The dots can be thought of as small lights mounted on the head, shoulders, elbows, hands, hips, knees, and feet of a person seen walking in profile. In the paradigm we will focus on, the dot person is seen as if walking in place on a treadmill, and the change that is to be detected is that the place in the walking cycle is either advanced or regressed from where it should be (either during a saccade or during a fixation). One way to think about this change is that the cycle of the walking dot person is a sequence of still snapshots that progress in sequence when there is not any change. However, when there is a change, the "film" is not moved to the next snapshot in the sequence but, instead, is moved to a "wrong" next snapshot that is a given distance in the sequence from the correct one. (However, after this jump in the sequence, the sequence then proceeds normally.) The size of the change was manipulated systematically (there were obviously no-change trials as well), and change detection was plotted as a function of the size of the change. The quite surprising finding was that detection of a change made during the saccade was quite good, although it was significantly worse than when the change was made during a fixation. However, detection of change across saccades was better than chance even for the smallest changes employed.

Similar findings were obtained when there was a change in orientation of the dot walker (i.e., the angular orientation of the walker with respect to the viewer). Here, as with 
the above change detection task, performance was only slightly worse when the change of orientation occurred during a saccade than when the change occurred during a fixation (Verfaillie \& De Graef, 2000; Verfaillie et al., 1994). Interestingly, the findings for these two types of changes contrasted with another condition in which the dot walker walked across the screen and the change that was to be detected was that the walker was either ahead of or behind where he or she should have been (again, the change occurred either during a saccade or during a fixation). Here again, detection performance was better than chance when the translation change was made during the saccade, but it was almost an order of magnitude worse than when the translation change was made during a fixation. Performance got somewhat better in this task, however, when there were companion dot walkers (Verfaillie, 1997). This difference in performance between detecting location changes and rotation changes is somewhat surprising, since one might think that memory for where something is should be more important to preserve than memory for what place something is in a cycle such as walking.

We should note that in all their experiments, Verfaillie and co-workers (Verfaillie, 1997; Verfaillie \& DeGraef, 2000; Verfaillie et al., 1994) employed a manipulation that would prevent detection of these changes by noticing that there was rapid motion on the retina. The manipulation was to have the figure make a large move to a new configuration and/or location for one frame in all conditions (i.e., in both change and no-change conditions) and then either return to where it "should have been" or to a changed location or configuration. Thus, rapid motion occurred both in change conditions and in no-change conditions. This manipulation was designed both to ensure that detection of changes that occurred during a saccade were not due to detection of rapid motion and to help equate the within-fixation and within-saccade change conditions, because detection of such rapid motion by low-level motion detectors in the visual cortex is undoubtedly much easier during a fixation.

The work of Verfaillie and colleagues thus presents a different picture than does most of the other work on integration of information across fixations, in that it clearly indicates that participants are quite good at integrating certain kinds of information about biological motion (or at least detecting changes in biological motion) across fixations. Moreover, it is unlikely that this change detection is due to verbal coding (or other coding schemes that are not likely to be part of normal visual perception). ${ }^{4}$ These experiments raise the question of whether there is something unique about biological motion, perhaps owing to the adaptive significance of perceiving the details of such motion. The present experiments probe the question of whether motion information can be integrated well across fixations, employing a paradigm similar to Verfaillie's, but using simple nonbiologicalmotion-specifically, simple (constant velocity) rotary motion of a simple object (a line). As with Verfaillie's work, we varied the size of the jump in motion that occurred during a saccade and employed a within-fixation jump condition as a baseline. However, the manipulation we used to control for detection of motion during the saccade was somewhat different from Verfaillie's.

\section{EXPERIMENT 1}

\section{Method}

Participants. The 12 participants were from the University of Massachusetts community, and all had normal uncorrected vision. They were paid for their participation.

Apparatus. Eye movements were recorded by a Generation V Fourward Technologies Dual Purkinje Eyetracker, which has a resolution of less than $10 \mathrm{~min}$ of arc, and the output was linear over the angles subtended by the displays used. The eyetracker was interfaced with a Megatek Whizzard vector-graphics display with a P-31 phosphor. The position of the eye was sampled every millisecond, and each $4 \mathrm{msec}$ of eyetracker output was compared with the output of the previous $4 \mathrm{msec}$ to determine whether the eyes were fixed or moving. The computer was also interfaced with a Megatek vector display monitor, on which the stimuli were presented. For the size of the displays plotted, the refresh rate of the display was $2.5 \mathrm{msec}$. The display was $91 \mathrm{~cm}$ from the participant's eye, and 120 pixels equalled $0.45^{\circ}$ of visual angle. Viewing was binocular, but eye movements were recorded from the participant's right eye. A bitebar was used to eliminate head movements during the experiment. ${ }^{5}$

Stimuli and Design. The experimental stimuli were rotating lines that were 1,200 pixels long and subtended $4.5^{\circ}$ of visual angle. The actual stimulus presentation is perhaps most clearly described by thinking about a trial as a succession of frames, with each frame lasting $15 \mathrm{msec}$. (During each frame, the line was "turned on" only during the first $5 \mathrm{msec}$, in order to minimize screen afterimages.) During normal rotation, the line presented in frame $n+1$ was rotated $2.5^{\circ}$ from the line presented in frame $n$. This created an excellent simulation of continuous rotational motion at a moderate speed, with a complete rotation of the line taking $2.16 \mathrm{sec}$.

At the beginning of each trial, a check calibration pattern came on with five fixed target crosses (one in the center of the screen, and the other four to the right, left, above, and below the center cross) and a calibration cross that moved in synchrony with the eye. If, while fixating one of the target crosses, there was a discrepancy between the calibration cross and the fixed cross, the eyetracker was recalibrated. If the calibration was accurate, the participant was asked to refixate the cross in the center of the display. When the center cross was fixated, the experimenter then triggered the trial by presenting the rotating line, which was rotating around the fixation point. After $500 \mathrm{msec}$, a second cross appeared, randomly to either the right or the left of the initial fixation point, which was a signal to fixate the second cross as soon as possible. The second cross was 1,000 pixels $\left(3.75^{\circ}\right)$ distant from the first cross. On one third of the trials, the line simply rotated at a constant rate throughout the trial. On the other two thirds of the trials, however, the line jumped at some point. That is, the line presented in frame $n+1$ was not one $2.5^{\circ}$ step ahead of the line presented in frame $n$. Instead, it was $5,10,15$, or 20 steps (i.e., $12.5^{\circ}, 25^{\circ}, 37.5^{\circ}$, or $50^{\circ}$ ) either ahead or behind where it "should have been" if motion was continuous. However, after the jump, the rotation resumed as it had previously, with a $2.5^{\circ}$ move each $15 \mathrm{msec}$. On the trials in which the line jumped, one third of them were before there was a saccade on the trial, one third were during the saccade, and the other third were after the end of the saccade. The jumps before the saccade were triggered $100 \mathrm{msec}$ after the cross appeared, which was before any saccade started; the jumps during the saccade were triggered $10 \mathrm{msec}$ after the beginning of the saccade was detected; and the jumps after the saccade were triggered $100 \mathrm{msec}$ after the end of the saccade was detected. The computa- 
tion of the event plus the refresh rate of the screen was less than $3 \mathrm{msec}$, which means that the actual display change was within $3 \mathrm{msec}$ of the time of the nominal change above.

At the end of each trial, the participants were asked to make two judgments, neither of which was timed. The first was to judge whether there had been a jump of the line during the trial, and the second was to judge whether the jump was forward or backward. The latter was a forced-choice judgment, so that they were told to make a forwardbackward judgment even if they had judged that there had been no jump. The forward-backward judgment was added to determine whether the participants had a reasonably veridical percept of the jump or merely noticed that "something funny happened."

Each participant saw a total of 360 trials, half on Day 1 and half on Day 2. There were 10 trials per participant in each of the 24 jump conditions. (The program arbitrarily designated 40 of the no-jump trials to the before saccade condition, 40 to the across-fixation condition, and 40 to the after-saccade condition.) On each day, exactly half the total number of trials in each condition was presented, and the order of the trials was randomized separately on each day. On Day 1, there were 10 practice trials in which the participants got feedback as to whether there was a display change or not, followed by 10 practice trials run just like the experimental trials. On Day 2, the experimental trials were preceded only by 10 practice trials run just like the experimental trials.

Procedure. When a participant arrived for the experiment, a bitebar was prepared and the eyetracking system was calibrated. The calibration period usually lasted less than $5 \mathrm{~min}$. After the calibration was completed, the purpose of the experiment was explained to the participant. On each trial, the calibration procedure was checked by having the participant fixate several boxes. If the calibration was satisfactory, he or she was instructed to fixate the center box, which was where the center of the rotating line would appear, and a trial began. If the calibration was not satisfactory, the eyetracker was recalibrated.

\section{Results and Discussion}

First, consider the results from the yes-no judgments (i.e., "Was there a jump?"). The principal result was that the participants were quite able to detect the jumps of the moving line, even when the jumps occurred during the saccade (see Table 1). Even at the smallest jump size $\left(12.5^{\circ}\right)$, detection of a jump during the saccade was significantly better than the false alarm rate $[t(11)=2.53$, $p=.03$, and $t(11)=4.15, p=.002$, for the forward and the backward jumps, respectively]. Detection of the jump during the saccade, however, was much worse than detection of the jump during a fixation $(23 \%$ vs. $80 \%)$ for the smallest jump size $[t(11)=14.9, p<.001]$. The false alarm rates in all three no-change conditions (which were physically identical) were $10 \%-11 \%$. (For convenience, we will refer to the conditions in which jumps occurred during fixations as the within-fixation conditions and the conditions in which the jumps occurred during the sac- cade as the across-fixation conditions, and we will we focus on the difference between the across-fixation conditions and the average of the two within-fixation conditions, since there did not seem to be any dramatic differences between the latter two conditions.) For the three larger jump sizes, the difference in performance between the across-fixation and the within-fixation conditions narrowed considerably: $63 \%$ versus $97 \%, 83 \%$ versus $97 \%$, and $93 \%$ versus $97 \%$, for $25^{\circ}, 37.5^{\circ}$, and $50^{\circ}$ jumps, respectively, although the difference was significant for all jump sizes $[t(11)=6.18, p<.001, t(11)=6.87, p<$ .001 , and $t(11)=2.96, p=.01$, respectively].

Next consider the forward-backward judgments (see Table 2). It appears that the participants almost always responded "backward" when they decided that there was not a jump (see the no-jump conditions), so that it is probably most useful to take the difference between the percentage of forward judgments in the forward and the backward jump conditions of the same size to assess how well the participants judged the direction of the jump at various jump sizes. In the across-fixation condition, the difference in the percentage of forward judgments in the forward and backward jump conditions was only $7.7 \%[t(11)=1.35$, $p=.20]$ at the smallest jump size $\left(12.5^{\circ}\right)$. At the three larger jump sizes in the across-fixation condition, however, there was a significant discrimination between the forward and backward jumps. The differences in the percent of forward responses between forward and backward jump conditions were $30 \%[t(11)=5.61, p<.001], 37 \%$, $[t(11)=4.12, p=.002]$, and $47 \%[t(11)=7.91, p<.001]$, in the $25^{\circ}, 37.5^{\circ}$, and $50^{\circ}$ jump conditions, respectively.

The participants thus detected the direction of the jump significantly above chance when the jump occurred during the saccade for all but the smallest jump size, indicating not only that they were detecting that "something funny happened," but also that they perceived the jump reasonably accurately. However, as with the yes-no judgments, detection of the direction of jump was quite a bit better in the within-fixation conditions than in the acrossfixation conditions. That is, averaging over the two withinfixation conditions, the differences between the percentage of forward judgments for the forward versus backward jump conditions were $33 \%, 68 \%, 69 \%$, and $57 \%$, for the $12.5^{\circ}, 25^{\circ}, 37.5^{\circ}$, and $50^{\circ}$ jumps, respectively $[t(11)=$ $3.83, p=.003, t(11)=10.5, p<.001, t(11)=10.7, p<$ .001 , and $t(11)=9.5, p<.001$, respectively], which was significantly better discrimination than in the acrossfixation conditions for all but the biggest jump size

Table 1

Experiment 1: Probability of Yes (It Jumped) Judgments

\begin{tabular}{lrrrrrrrrr}
\hline & \multicolumn{7}{c}{ Size and Direction of Jump } \\
\cline { 2 - 10 } \multicolumn{1}{c}{ Interval } & $+50^{\circ}$ & $+37.5^{\circ}$ & $+25^{\circ}$ & $+12.5^{\circ}$ & No Jump & $-12.5^{\circ}$ & $-25^{\circ}$ & $-37.5^{\circ}$ & $-50^{\circ}$ \\
\hline Before saccade & .983 & .985 & .983 & .808 & .110 & .910 & .942 & .951 & .975 \\
Across fixations & .923 & .883 & .625 & .208 & .096 & .250 & .626 & .767 & .932 \\
After saccade & .975 & .975 & .967 & .581 & .088 & .892 & .983 & .974 & .951 \\
\hline
\end{tabular}


Table 2

Experiment 1: Probability of Forward (It Jumped Forward) Judgments

\begin{tabular}{lrrrrrrrrr}
\hline & \multicolumn{7}{c}{ Size and Direction of Jump } \\
\cline { 2 - 10 } \multicolumn{1}{c}{ Interval } & $+50^{\circ}$ & $+37.5^{\circ}$ & $+25^{\circ}$ & $+12.5^{\circ}$ & No Jump & $-12.5^{\circ}$ & $-25^{\circ}$ & $-37.5^{\circ}$ & $-50^{\circ}$ \\
\hline Before saccade & .739 & .854 & .875 & .683 & .114 & .302 & .225 & .223 & .183 \\
Across fixations & .580 & .550 & .450 & .199 & .106 & .123 & .150 & .181 & .109 \\
After saccade & .705 & .883 & .887 & .533 & .098 & .258 & .182 & .144 & .116 \\
\hline
\end{tabular}

$[F(1,11)=16.98, p=.002, F(1,11)=49.29, p<.001$, $F(1,11)=21.01, p<.001$, and $F(1,11)=2.486, p=.14$, for the $12.5^{\circ} 25^{\circ}, 37.5^{\circ}$, and $50^{\circ}$ jumps, respectively].

In sum, the data indicate that visual information about the rotation of the line is preserved across the saccade: Detection of a jump was significantly better than chance in all conditions, and detection of whether the jump was forward or backward was significantly better than chance in all but the smallest jump condition. Nonetheless, performance was significantly better when the jump occurred during a fixation. This pattern of data raises the related questions of (1) whether there was an artifact producing performance well above chance when the jump occurred during the saccade and (2) whether there was an artifact producing the better performance when the jump was during a fixation. Of course, the word artifact is a loaded one, since it suggests a priori that a particular explanation for an effect is uninteresting, but the question is still worth exploring.

The answer to both questions is that there may, in fact, be a similar artifact. That is, at the point of the jump, there is a very rapid change of location for much of the rotating line that could trigger neurons that are sensitive to large velocities on the retina. Although it does not seem all that plausible that such large velocities could be detected during a saccade (when virtually the whole visual world is undergoing large velocity changes on the retina and there is saccadic suppression), it is not impossible. For the jumps during the fixation, on the other hand, it seems likely that detection of these velocities are part of why performance was better when the jump was during the fixation than when it was during the saccade. However, it is then an open question as to whether this is an artifact or a natural part of the phenomenon of detecting a jump. Nonetheless, even in the during-fixation conditions, it would be of interest to determine how much of the detection of a jump is due to detecting these high-velocity movements and how much of the detection of the jump is due to detecting that the line is not where it should be. Experiment 2 was designed to help answer these questions.

\section{EXPERIMENT 2}

Experiment 2 was a replication of Experiment 1, with one major change in procedure designed to eliminate the likelihood that the discontinuity in the motion of the line could be detected by detecting the event of the jump via sensing a high-velocity movement on the retina. To achieve that end, on all trials, there were two masking frames in the rotation cycle (i.e., a period of $30 \mathrm{msec}$ ) in which all the lines representing the rotating line were displayed simultaneously. That is, in each of these two frames, all the 144 lines that represented the rotating line were displayed for $5 \mathrm{msec}$, followed by $10 \mathrm{msec}$ of off time. In the no-jump condition, the line appeared where it should have been after these two frames. That is, if the line was in position $n$ before the two masking frames, it would appear in position $n+3$ after the masking frames. In the jump conditions, the line would be altered the appropriate angular distance from position $n+3$. Other than that, the experimental conditions and procedure were identical to those in Experiment 1. One consequence of this change, which made the design of this experiment different from that of Experiment 1, was that the no-jump conditions were no longer identical: For one third of them, the two masking frames occurred during the first fixation (but no jump); for one third, the two masking frames occurred primarily during the saccade; and for the other third, the two masking frames occurred during the second fixation.

\section{Method}

Participants. The 8 participants were from the University of Massachusetts community, and all had normal uncorrected vision. They were paid for their participation.

Apparatus and Procedure. The apparatus and procedure were the same as those in Experiment 1 . The only difference was that the participants were warned about the mask that would appear on each trial and were told to ignore it as best they could.

Stimuli and Design. The stimuli and design were the same as those in Experiment 1, except for the two intervals on which the masking display occurred. As with all the other intervals during the trial, the masking stimulus was displayed for $5 \mathrm{msec}$, followed by a 10-msec off period. However, during the two masking cycles, all 144 lines were displayed, rather than just 1 . The first of the two successive 15 -msec masking cycles was triggered $100 \mathrm{msec}$ after the cross appeared (in the before-saccade condition), $10 \mathrm{msec}$ after the beginning of the saccade was detected (in the within-saccade condition), or $100 \mathrm{msec}$ after the end of the saccade was detected (in the after-saccade condition). After the masking frames, on one third of the trials, the line appeared where it should have been after $45 \mathrm{msec}$ (at location $n+3$ ). On the other two thirds of the trials, it was displaced from that rotational angle by plus or minus $12.5^{\circ}, 25^{\circ}, 37.5^{\circ}$, or $50^{\circ}$ (as in Experiment 1). As was indicated above, however, the no-jump trials were now different from each other, since the mask appeared on those trials before, during, or after the saccade. It is possible, however, that the masking frames in the within-saccade (or across-fixation) condition were not always terminated before the second fixation began. That is, the on portion of the second of these two frames ended $20 \mathrm{msec}$ after the signal to trigger the display or as much as $33 \mathrm{msec}$ after the saccade began. Moreover, there was noticeable phosphor persistence from this masking display (which, however, clearly masked any persistence from the prior rotating 
line). Nonetheless, the important fact is that the rotational information that was needed to make the judgment of jump versus no jump in these conditions came from before and after the saccade (i.e., on two separate fixations).

As in Experiment 1, the participants were asked to make two judgments at the end of each trial: (1) whether there had been a jump of the line during the trial and (2) whether the jump was forward or backward. As in Experiment 1, each participant saw a total of 360 trials, half on Day 1 and half on Day 2. There were thus 10 trials per subject in each of the 24 jump conditions, and 40 in each of the nojump conditions. The rest of the procedure was also the same as that in Experiment 1.

\section{Results and Discussion}

One striking aspect of the data (see Table 3 ) was that the false alarm rates were much higher in the two withinfixation conditions than in the across-fixation condition. However, even the false alarm rates in the across-fixation condition were noticeably higher than those in Experiment 1. It thus seems as though the participants had a hard time dissociating the masking event from a jump. When the mask occurred during a fixation, it was particularly salient and appeared to trigger a "yes, it jumped" response even when there was no jump. Although the mask was less salient when it was triggered during the saccade, its afterimage was often still perceptable and appeared to trigger some "yes, it jumped" responses. As a result of these differing false alarm rates, it is somewhat harder to compare the detection rates for the jump in the across-fixation condition with those in the within-fixation conditions, but $d^{\prime}$ and $A^{\prime}$ measures are two possible ways to do so. We will first consider detection performance as the difference between the probability of a yes judgment in a jump condition and the probability of a yes judgment in the appropriate no-jump control and then will report $d^{\prime}$ and $A^{\prime}$ results.

When the forward and the backward jump conditions were averaged, the differences in percentage of yes judgments between the jump and the no-jump conditions for the across-fixation conditions were $2 \%(t<1), 10 \%$ $[t(7)=1.74, p>.10], 25 \%[t(7)=5.34, p<.001]$, and $39 \%[t(7)=6.25, p<.001]$, in the $12.5^{\circ}, 25^{\circ}, 37.5^{\circ}$, and $50^{\circ}$ jump conditions, respectively. It thus appears that discrimination is good in the two larger jump conditions, and there is a suggestion of some appreciable discrimination in the $25^{\circ}$ conditions. Moreover, discrimination was no better in the within-fixation conditions. Averaging over the before-saccade and after-saccade conditions and averaging over forward and backward jumps, differences in percentage of yes judgments between the jump and the nojump conditions were $0 \%(t<1), 8 \%[t(7)=2.23, p=$ $.06], 12 \%[t(7)=1.92, p=.09]$, and $21 \%[t(7)=5.15$, $p<.001]$. Thus, by this difference measure, detection of the jump actually appeared to be slightly better in the across-fixation conditions than in the during-fixation conditions. On average, over all jump sizes, the difference in percentage of yes judgments between the jump and the nojump conditions was $19 \%$ in the across-fixation jump conditions $[t(7)=4.95, p=.002]$ but only $10 \%$ in the withinfixation conditions $[t(7)=2.74, p=.03]$. The $9 \%$ difference between these two differences was only marginally significant, however $[F(1,7)=4.94, p=.06]$.

Now let us consider the relative detection performance in the within-fixation and across-fixation conditions, using $d^{\prime}$ and $A^{\prime}$. The basic pattern was the same as that in the above analysis, with only slightly different details. To minimize noise and to eliminate cells with probabilities of 0 or 1 , for each jump size, we averaged data over the forward and the backward conditions, and for the withinfixation conditions, we also averaged over the fixation on which the jump and/or mask occurred. For the four jump sizes (going from smallest to biggest), the mean $d$ 's in the across-fixation conditions were $0.04,0.27,0.69$, and 1.1 $[t(7)=0.52,1.81,5.29$, and $5.99, p \mathrm{~s}>.2,>.1,<.001$, and $<.001$, respectively]. The analogous values for the within-fixation conditions were $.01, .22, .39$, and .78 $[t(7)=0.06, p<.2, t(2)=2.23, p<.1, t(7)=2.06, p<$ .1 , and $t(7)=5.59, p<.001$, respectively]. The difference between the across-fixation and within-fixation conditions was significant only for the largest jump size $[t(7)=2.59, p=.04]$. Both the discriminability and the difference between conditions looked slightly less impressive using $A^{\prime}$ as the measure. For the across-fixation conditions, $A^{\prime}$ was $.505, .546, .699$, and $.806[t(7)=0.14$, $p<.2, t(7)=0.77, p>.2, t(7)=7.48, p<.001$, and $t(7)=10.5, p<.001$, respectively], and for the withinfixation conditions, it was $.420, .590, .598$, and .817 ( $p$ s $>$ $.2,<.2,>.2$, and $<.001$, respectively). With the $A^{\prime}$ measure, none of the differences between the across-fixation and the within-fixation conditions was close to significant (all $t \mathrm{~s}<1$ ).

Thus, as was indicated above, the $d^{\prime}$ and $A^{\prime}$ analyses mirrored those using the raw probability values. The only difference was that, using the $d^{\prime}$ analysis, there was some indication of an actual superiority of the across-fixation conditions to the within-fixation conditions. Even though there was no baseline problem in Experiment 1, we thought it would be instructive to compute the analogous $d$ 's and $A$ 's to compare the across-fixation and the within-fixation conditions. Because there were many cells in which the hit rate was 1.0 and the false alarm rate was zero, a $d^{\prime}$ analysis in each jump condition was not possible. When

Table 3

Experiment 2: Probability of Yes (It Jumped) Judgments

\begin{tabular}{lccccccccc}
\hline & \multicolumn{7}{c}{ Size and Direction of Jump } \\
\cline { 2 - 9 } \multicolumn{1}{c}{ Interval } & $+50^{\circ}$ & $+37.5^{\circ}$ & $+25^{\circ}$ & $+12.5^{\circ}$ & No Jump & $-12.5^{\circ}$ & $-25^{\circ}$ & $-37.5^{\circ}$ & $-50^{\circ}$ \\
\hline Before saccade & .900 & .893 & .875 & .700 & .693 & .775 & .750 & .788 & .925 \\
Across fixations & .824 & .608 & .450 & .375 & .384 & .425 & .513 & .663 & .713 \\
After saccade & .875 & .750 & .763 & .600 & .691 & .688 & .688 & .888 & .888 \\
\hline
\end{tabular}


one first averages over all jump conditions and then computes $d^{\prime}$, it was 2.05 and 2.96 in the across-fixation and within-fixation conditions $[t(11)=13.8$, and 19.0, respectively, both $p \mathrm{~s}<.001$ ], and the $d^{\prime}$ in the within-fixation condition was significantly bigger than that in the acrossfixation condition $[t(11)=6.76, p<.001] .{ }^{6} A^{\prime}$ in the four jump conditions (going from smallest to biggest) was $.612, .856, .933$, and .979 in the across-fixation conditions $[t(11)=3.96,10.1,29.1$, and $54.9, p s<.002$, respectively] and $.936, .998, .995$, and .994 in the within-fixation conditions $[t(11)=17.8,69.5,96.3$, and $109.4, p \mathrm{~s}<$ $.001] . A^{\prime}$ in the within-fixation condition was significantly bigger than in the across-fixation condition for each jump size $[t(11)=14.9,6.28,6.82$, and $2.83, p$ s $<.02]$.

Discrimination of whether the jump moved forward or backward was not very good in either the across-fixation or the within-fixation conditions (see Table 4). Overall, in the within-fixation conditions, there were $41 \%$ forward judgments in the forward jump conditions, as compared with $32 \%$ forward judgments in the reverse jump conditions $[t(7)=2.11, p=.07]$. Discrimination was even worse in the across-fixation conditions, with $26 \%$ forward judgments in the forward jump conditions, as compared with $22 \%$ forward judgments in the reverse jump conditions $[t(7)=1.17, p>.20]$. Even in the biggest withinfixation jump conditions (plus and minus $50^{\circ}$ ), discrimination was not all that impressive. For the within-fixation conditions, there were $53 \%$ forward judgments in the forward jump conditions, as compared with $33 \%$ forward judgments in the reverse jump conditions $[t(7)=1.70$, $p=.13$ ], and in the across-fixation conditions, there were $41 \%$ forward judgments in the forward jump conditions, as compared with $23 \%$ forward judgments in the reverse jump conditions $[t(7)=2.41, p=.05]$.

It thus appeared that the presence of the masking interval interfered quite a bit with the directional judgments. Part of the problem may have been that the participants reacted "yes, there was a jump" upon seeing the mask (whether or not there was a jump) and may have typically encoded that as either a forward or a backward judgment (and the bias may have been different for different participants).

\section{GENERAL DISCUSSION}

The yes-no data of Experiment 2 complement those of Experiment 1 by indicating that detection of a jump across fixations was significantly above chance. These data thus indicate that the visual system can actually track rotary motion across fixations, rather than just making the default assumption that rotary motion is smoothly continu- ing across the saccadic interval. However, as opposed to Experiment 1, detection of a jump in Experiment 2 was significantly above chance only for the two larger jump sizes. Moreover, discrimination of whether the jump was forward or backward was substantially worse in both the acrossfixation and the within-fixation conditions of Experiment 2 than in those of Experiment 1.

There are two possible explanations for this difference in performance between the two experiments. The first is that a substantial part (although not all) of the good discrimination performance in the across-fixation conditions of Experiment 1 was due to actually detecting the jump (or detecting motion) during the saccade despite (1) saccadic suppression, (2) the other rapid large velocities appearing on the retina during the saccade, and (3) postsaccadic suppression. The second is that the substantially poorer performance in Experiment 2 was due to seeing the mask during the saccade (as a massive increase in brightness) and/ or to seeing its afterimage at the end of the saccade and then having a difficult time determining whether other changes occurred in the situation in addition to this disruptive event. We think that the second explanation is probably nearer to the truth, chiefly because performance in the within-fixation conditions in Experiment 2 was actually worse than that in the across-fixation conditions (although not significantly), which was likely due to the even greater disruption of seeing the mask fully during a fixation. A second reason that we are skeptical that the good performance in the across-fixation condition was completely due to an artifact of seeing some motion during the saccade is that the forward-backward judgments in Experiment 1 in the across-fixation condition were quite good as well. Although it is not implausible that there might be detection of some sort of rapid motion during a saccade, it seems much less plausible that the direction of the motion would be accurately recorded on top of a background of large retinal velocities on the saccading eye in the whole array.

In sum, the present experiments indicate that information about rotary motion of a line is preserved across a saccade and integrated quite well with the rotary information observed on the succeeding fixation. Moreover, our findings are consonant with the findings of Verfaillie and co-workers (Verfaillie, 1997; Verfaillie \& De Graef, 2000; Verfaillie et al., 1994), indicating that both simple rotary motion and more complex biological motion are preserved quite well across fixations. Thus, we think that it is likely that the results of Verfaillie and colleagues reveal something about the perception of something like rotary motion in general, rather than something special about bi-

Table 4

Experiment 2: Probability of Forward (It Jumped Forward) Judgments

\begin{tabular}{lccccccccc}
\hline & \multicolumn{7}{c}{ Size and Direction of Jump } \\
\cline { 2 - 9 } \multicolumn{1}{c}{ Interval } & $+50^{\circ}$ & $+37.5^{\circ}$ & $+25^{\circ}$ & $+12.5^{\circ}$ & No Jump & $-12.5^{\circ}$ & $-25^{\circ}$ & $-37.5^{\circ}$ & $-50^{\circ}$ \\
\hline Before saccade & .500 & .438 & .425 & .350 & .254 & .288 & .425 & .300 & .375 \\
Across fixations & .407 & .253 & .238 & .138 & .156 & .163 & .213 & .263 & .225 \\
After saccade & .563 & .425 & .338 & .275 & .247 & .238 & .288 & .350 & .288 \\
\hline
\end{tabular}


ological motion. Of course, it could be that this capability developed in humans (and other species) because of the importance of perceiving biological motion veridically. However, even if this is true, the apparatus developed primarily for that purpose is not limited to the perception of biological motion.

We do feel that the present paradigm, although a change detection paradigm, gets at how motion information is typically processed in normal viewing. When a change is detected, one's introspective experience is that it is a visual phenomenon, rather than the result of problem solving or deduction. That is, we think that it gets at how visual information about moving objects is typically integrated across fixations in normal viewing. Because people are unaware of the blanking during saccades and because saccades are of varying durations, it would seem quite improbable that people could figure out whether the line jumped during the saccade as a conscious problem-solving activity. They would have to know where the line was when the saccade began and know how long the saccade lasted and then compute, from those two pieces of information, where it would land. Instead, it appears that the visual system is doing something like this computation for them automatically.

Relating these findings of detection of motion changes to detection of changes in static information across fixations is not straightforward. Changes in both moving and fixed objects can be detected across fixations if they are big enough. However, evaluating whether the sensitivity of the system to change is similar in the two situations is close to impossible, because the dimensions of change are different. One way to compare motion changes to changes in static information is to compare performance in withinfixation changes with that in between-fixation changes. Henderson (1997) made this comparison for a static display: a line drawing of an object that appeared to be behind something like a "picket fence." There were several types of changes in the display, but the one of most relevance shifted the whole display one "picket" unit. In the withinfixation condition, the change was detected $81 \%$ of the time, with a false alarm rate of $4 \%$, whereas in the betweenfixation condition, detection of change was $26 \%$, with a false alarm rate of $21 \%$. As compared with performance in Experiment 1 at the smallest jump size, the performance in Henderson's within-fixation condition was somewhat better (the false alarm rate was lower), but performance in his between-fixation condition was worse. This is despite the fact that in his within-fixation condition, both views were a large distance from the fovea, whereas ours were right at fixation. If Henderson's finding is typical of static displays, it would suggest a difference between the integration of static and dynamic information across fixations, especially since we observed no difference between accuracy in detecting within-fixation and between-fixation changes in Experiment 2 when motion cues during a fixation were eliminated.

If one accepts that our experiments get at integration of visual information across fixations, however, we think that the conclusions differ quite a bit from other paradigms that have tried to get at integration. Certainly, in the studies dealing with reading, participants are unaware of and are unaffected by reasonably large changes in the surface form of the information (e.g., case changes of all letters). Moreover, even when naming latencies or fixation durations are affected by changes across fixations (e.g., as by changes in the meanings of words from fixation to fixation), participants are virtually never aware that these changes take place, even though the changed word is fixated immediately after the change. Furthermore, as we argued above, although the studies in which object identification across fixations was examined showed some effects related to surface visual changes (e.g., a smaller preview benefit when the object's mirror image or a rotated version of the object was the preview), other surface changes (e.g., change in size) had no effect. It thus appears that dynamic visual information might be better preserved across fixations than is static visual information. One possible ecological reason for this is that most dynamic acts we seeboth biological motion, such as running, and other motion, such as the trajectory of a moving object—are extended in time (well over a typical fixation duration), and in order to make sense of them, we often need to integrate detailed information about the dynamic process across several fixations. In contrast, when viewing a static scene, there is little visual information that needs to be preserved across fixations that can help in understanding the scene better. Even in cases in which integrating details of visual information from fixation to fixation might help, such as computing the layout of a scene, the human visual apparatus apparently does not do it, as is evidenced by the difficulty in recognizing the absurdity of "impossible scenes" and "impossible objects," such as Escher's ever-ascending staircase or the impossible tuning fork.

In conclusion, in two experiments, we found that participants performed significantly better than chance in detecting displacements (or jumps) in simple rotary motion. Moreover, in Experiment 2, when perception of information about very rapid motion was suppressed by a mask, perception of the jump was actually slightly worse when it occurred during a fixation than when it occurred across fixations. These results complement and extend the findings of Verfaillie and co-workers, who found that perception of changes in biological motion was quite good when the change occurred across fixations. The data also indicate that relatively low level visual motion information can be integrated across fixations and are in contrast to findings that indicate that some changes in visual properties across fixations can have little influence on how objects are recognized.

\section{REFERENCES}

Bridgeman, B., Hendry, D., \& Stark, L. (1975). Failure to detect displacement of the visual world during saccadic eye movements. Vision Research, 15, 719-722.

Carlson-Radvansky, L. A. (1999). Memory for relational information across eye movements. Perception \& Psychophysics, 61, 919-934.

Carlson-Radvansky, L. A., \& Irwin, D. E. (1995). Memory for structural information across eye movements. Journal of Experimental Psychology: Learning, Memory, \& Cognition, 21, 1441-1458.

Currie, C. B., McConkie, G. W., Carlson-Radvansky, L. A., \& 
IRWIN, D. E. (2000). The role of the saccade target object in the perception of a visually stable world. Perception \& Psychophysics, 62, 673-683.

Hayhoe, M. [M.], Lachter, J., \& Feldman, J. (1991). Integration of form across saccadic eye movements. Perception, 20, 393-402.

Henderson, J. M. (1997). Transsaccadic memory and integration during real-world object perception. Psychological Science, 8, 51-59.

Henderson, J. M., \& Hollingworth, A. (1998). Eye movements during scene viewing: An overview. In G. Underwood (Ed.), Eye guidance in reading and scene perception (pp. 269-294). Amsterdam: Elsevier.

Henderson, J. M., \& Hollingworth, A. (1999). The role of fixation position in detecting scene changes across saccades. Psychological Science, 10, 438-443.

Henderson, J. M., Pollatsek, A., \& Rayner, K. (1987). The effects of foveal priming and extrafoveal preview on object integration. Journal of Experimental Psychology: Human Perception \& Performance, 13, 449-463.

Henderson, J. M., \& Siefert, A. B. C. (1999). The influence of enantiomorphic transformation on transsaccadic object integration. Journal of Experimental Psychology: Human Perception \& Performance, 25, 243-255.

IRWIN, D. E. (1992). Visual memory within and across fixations. In $\mathrm{K}$. Rayner (Ed.), Eye movements and visual cognition: Scene perception and reading (pp. 146-165). New York: Springer-Verlag.

Irwin, D. E., McConkie, G. W., Carlson-Radvansky, L., \& Currie, C. (1994). A localist solution for visual stability across saccades. Behavioral \& Brain Sciences, 17, 265-266.

IRwIN, D. E., YANTIS, S., \& JoNIDES, J. (1983). Evidence against visual integration across saccadic eye movements. Perception \& Psychophysics, 34, 49-57.

Li, W., \& Matin, L. (1990). Saccadic suppression of displacement: Influence of postsaccadic exposure duration and of saccadic stimulus elimination. Vision Research, 30, 945-955.

MACK, A. (1970). An investigation of the relationship between eye and retinal image movement in the perception of movement. Perception \& Psychophysics, 8, 291-298.

McConkIE, G. W., \& Currie, C. B. (1996). Visual stability across saccades while viewing complex pictures. Journal of Experimental Psychology: Human Perception \& Performance, 22, 563-581.

McConKIE, G. W., \& ZoLA, D. (1979). Is visual information integrated across successive fixations in reading? Perception \& Psychophysics, 25, 221-224.

McConkie, G. W., Zola, D., \& Wolverton, G. S. (1980, April). How precise is eye guidance in reading? Paper presented at the American Educational Research Association Meeting, Boston.

Morris, R. K., Rayner, K., \& Pollatsek, A. (1990). Eye movement guidance in reading: The role of parafoveal letter and space information. Journal of Experimental Psychology: Human Perception \& Performance, 16, 268-281.

Pollatsek, A., Lesch, M., Morris, R. K., \& Rayner, K. (1992). Phonological codes are used in integrating information across saccades in word identification and reading. Journal of Experimental Psychology: Human Perception \& Performance, 18, 148-162.

Pollatsek, A., Raney, G. E., LaGasse, L., \& Rayner, K. (1993). The use of information below fixation in reading and in visual search. Canadian Journal of Experimental Psychology, 47, 179-200.

Pollatsek, A., \& Rayner, K. (1982). Eye movement control in reading: The role of word boundaries. Journal of Experimental Psychology: Human Perception \& Performance, 8, 817-833.

Pollatsek, A., \& Rayner, K. (1992). What is integrated across fixations? In K. Rayner (Ed.), Eye movements and visual cognition: Scene perception and reading (pp. 166-191). New York: Springer-Verlag.

Pollatsek, A., Rayner, K., \& Collins, W. E. (1984). Integrating pictorial information across eye movements. Journal of Experimental Psychology: General, 113, 426-442.
RAYNER, K. (1998). Eye movements in reading and information processing: 20 years of research. Psychological Bulletin, 124, 372-422.

RAYNer, K., McConkie, G. W., \& Zola, D. (1980). Integrating information across eye movements. Cognitive Psychology, 12, 206-226.

Rayner, K., \& Pollatsek, A. (1983). Is visual information integrated across saccades? Perception \& Psychophysics, 34, 39-48.

Rayner, K., Well, A. D., Pollatsek, A., \& Bertera, J. H. (1982). The availability of useful information to the right of fixation in reading. Perception \& Psychophysics, 31, 537-550.

Simons, D. J. (2000). Current approaches to change blindness. Visual Cognition, 7, 1-15.

Verfaillie, K. (1997). Transsaccadic memory for the egocentric and allocentric position of a biological-motion walker. Journal of Experimental Psychology: Learning, Memory, \& Cognition, 23, 739-760.

Verfaillie, K., \& De Graef, P. (2000). Transsaccadic memory for position and orientation of saccade source and target. Journal of Experimental Psychology: Human Perception \& Performance, 26, 12431259.

Verfaillie, K., De Troy, A., \& Van Rensbergen, J. (1994). Transsaccadic integration of biological motion. Journal of Experimental Psychology: Learning, Memory, \& Cognition, 20, 649-670.

\section{NOTES}

1. In addition to McConkie et al.'s (1980) research on detection of shifting the location of the text during a saccade, there is an extensive literature (see, e.g., Bridgeman, Hendry, \& Stark, 1975; Li \& Matin, 1990; Mack, 1970) on the detection of display displacements across eye movements in nonreading situations. Bridgeman et al. found that the target displacement could not be detected if the eye movement was more than three times larger than the target displacement. Thus, the results from using relatively simple visual stimuli are quite consistent with those from the reading literature.

2 . The participants were generally aware of display changes in these experiments.

3. We should note that the poor detection of change is not limited to changes across fixations, and in many circumstances in which there is some sort of interruption of viewing during a fixation, change detection is also often quite poor (see Simons, 2000, for an overview of this change blindness phenomenon).

4. The detection of a change in orientation might be somewhat easier to think of as being due to some sort of conscious coding mechanism (e.g., noting that the walker is at a $45^{\circ}$ angle to the viewer). However, one experiment (Verfaillie et al., 1994, Experiment 3) indicated that a change in orientation was still quite detectable when the dot walker was rotating around a vertical axis, although discrimination was somewhat worse than when the walker was at a fixed orientation before and after the change. It seems quite implausible to think of a conscious coding mechanism that could aid in detection of an orientation change for this continually rotating dot walker.

5. Since the experiment was run in a darkened room, the participants adapted to the dark during the experimental session. The luminance of the screen was adjusted periodically so that the stimuli (the rotation line and fixation crosses) were clearly visible but not subjectively bright enough to either cause the participant discomfort or to interfere with recording the eye movements. A fast decay (P31) phosphor was employed that quickly decays to within $1 \%$ of its original brightness. There is slower decay after that, so that there would be a dim residue of brightness of the stimuli displayed in the prior 200-300 msec.

6 . In all of these analyses, the average over all the no-jump conditions was used as the false alarm rate.

(Manuscript received September 7, 2000; revision accepted for publication January 18,2002 .) 